\title{
Investigation of the Shape Change of Bio-flocs and Its Influence on Mass Transport using Particle Image Velocimetry (PIV)
}

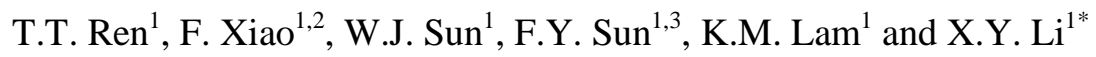 \\ ${ }^{1}$ Environmental Engineering Research Centre, Department of Civil Engineering, The University of \\ Hong Kong, Pokfulam Road, Hong Kong, China \\ ${ }^{2}$ Research Center for Eco-Environmental Sciences, Chinese Academy of Sciences, Beijing 100085, \\ China \\ ${ }^{3}$ Harbin Institute of Technology Shenzhen Graduate School, Shenzhen 518055, China \\ (*Email: xlia@hkucc.hku.hk)
}

\begin{abstract}
In this laboratory study, an advanced flow visualization technique - particle image velocimetry (PIV) - was employed to investigate the change of shape of activated sludge flocs in water and its influence on the material transport characteristics of the flocs. The continuous shape change of the bio-flocs that occurred within a very short period of time could be captured by the PIV system. The results demonstrate that the fluid turbulence caused the shift of parts of a floc from one side to the other in less than 200 milliseconds. During the continuous shape change, the liquid within the floc was forced out of the floc, which was then refilled with the liquid from the surrounding flow. For the bio-flocs saturated with a tracer dye, it was shown that the dye could be released out of the flocs at a faster rate when the flocs were swayed around in water. The experimental results indicate that frequent shape change of bio-flocs facilitates the exchange of fluid and materials between the floc interior and the surrounding water. This mass transfer mechanism can be more important than molecular diffusion and internal permeation to the function and behavior of particle aggregates, including bio-flocs, in natural waters and treatment systems.
\end{abstract}

Keywords

Bio-flocs, mass transfer, particle image velocimetry (PIV), permeability, shape change

\section{INTRODUCTION}

Most microorganisms in bioreactors in wastewater treatment are in the form of aggregates, or flocs. In many cases, as the bioflocs increase in size, mass transfer limitation has been regarded as one of the primary limiting factors to the treatment performance. Molecular diffusion is considered as the main mechanism of material transport into flocs (Clark, 2003). Recently, the importance of internal flow through aggregates to particle flocculation and mass transfer also has been recognized (Li et al., 2003). However, in almost all of the theoretical analyses and experimental studies, an aggregate has been treated as a rigid object with a fixed shape and volume. The soft and open structure of particle aggregates, including bio-flocs, has not been taken into account in description of their mass transport characteristics.

Research indicates that particle aggregates are fractals that have a self-similar, open and porous structure. This implies that, other than a rigid structure, aggregates suspended in water are rather soft and flexible in shape. In recent years, the phenomenon of shape change of biological aggregates in water has been recognized (Glasgow, 2003; Tao et al., 2006a, b). The deformation of bio-flocs during the compression of hydrodynamic forces has been observed (Glasgow, 2003). The shape change of activated sludge flocs was recorded during a freezing and thawing treatment process (Tao et al., 2006a, b). Shape change would occur when hydrodynamic forces act on the flocs in a sheared fluid flow, which would enhance the material exchange between the floc interior and the surrounding fluid. Thus, continual shape change could be another important mechanism of 
material transport for bio-flocs in water. However, the deformable feature of biological aggregates and its influence on mass transport have not been well addressed.

Particle image velocimetry (PIV) is a non-intrusive optical system that is able to capture the velocity vectors of a flow and the shape change of an object within a millisecond (Raffel et al., 2007). For various research purposes, the laser-based PIV technique has been applied to study the flow around bluff bodies, such as a circular cylinder oscillating in water (Lam and Dai, 2002) and an inclined flat plate (Lam and Leung, 2004). Flow patterns and quantitative flow information including instantaneous velocity vectors and streamlines can be obtained from the PIV images (Xiao et al., 2011; 2013). Therefore, in the present laboratory study, PIV technology was employed to investigate the change of shape of bio-flocs in a flow. The importance of continuous shape change and its effect on the material transport features of the flocs was also discussed.

\section{MATERIALS AND METHODS}

The shape change of bio-flocs in a flow field and related mass transfer features were investigated using a PIV system. The PIV setup consisted of a laser illumination, a high-speed CCD video camera and dedicated computing techniques to track particles for flow field illustration and velocity measurement (Xiao et al., 2011; 2013). In the present laboratory study, a rectangular glass chamber was used as a flow channel, for which a pump was used to control the flow direction. Polyamid seed particles $(\sim 5 \mu \mathrm{m})$ were suspended in water as the flow tracers in the chamber when needed. A fluorescent dye, Rhodamine WT, was used as a tracer dye for illustration of mass transfer from the flocs into the surrounding water.

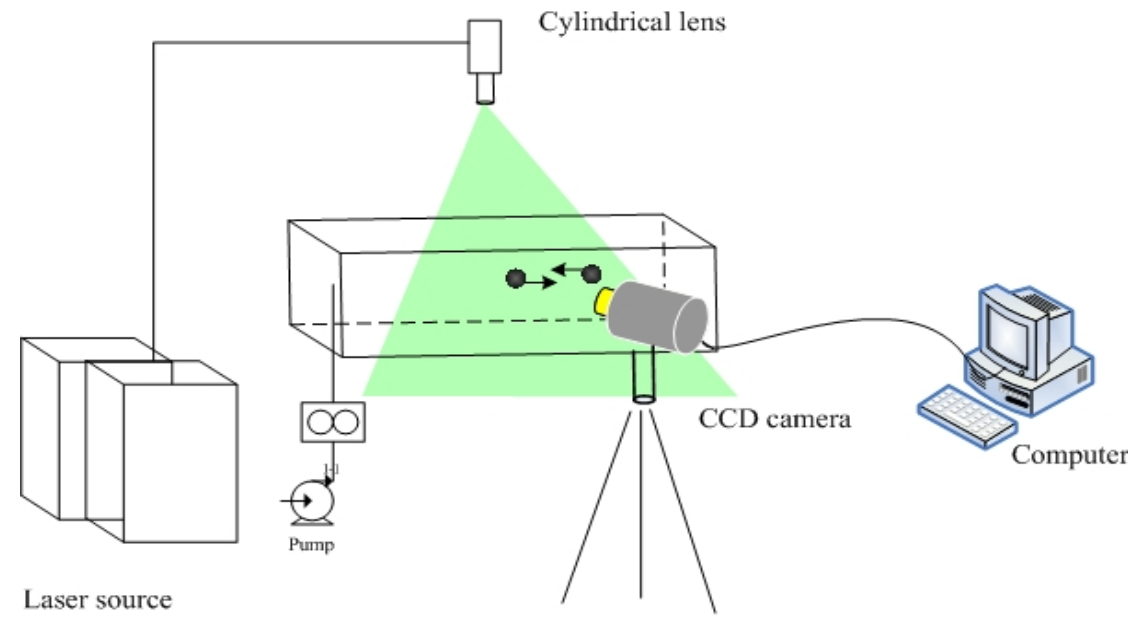

Fig. 1. Schematic of the PIV experimental setup.

The aggregates of activated sludge (AS) were produced on a jar-test device (ZR4-6, Zhongrun Co., Shenzhen, China) following the method described previously by Li et al. (2003). During a PIV test, a large activated sludge (AS) floc was fixed by glue at the tip of a needle. The floc was immerged gently into the water chamber. A laser beam was generated and expanded to a thin vertical laser sheet to illuminate the AS floc (Fig. 1). The planar laser sheet was illuminated through the centre of the floc to illustrate the details of the shape of the bio-floc and the flow around the floc. With a flow change, the floc would change its shape to conform the hydrodynamic force in the flow. The deformation of the bio-floc that occurred within a very short period of time was captured by the high-speed CCD camera (PCO.camera 1200, resolution $=1280 \times 1024$ pixels). The video images 
were stored in a computer and processed to determine the change of the shape of the floc in the flow and the resulting fluid exchange and material transport. The PIV tests were conducted for a number of AS flocs.

The mass transport feature of AS flocs was also investigated directly based on the rate of dye release from the flocs into water. Prior to the experimental tests, a certain amount of fluorescent Rhodamine WT was injected into the floc by a micro-syringe. A floc saturated with the tracer dye was then placed in water for the dye releasing tests. For the dye release from the AS flocs in a turbulent flow, a floc fixed at the needle tip was swayed gently in the water chamber (Fig. 2). The release of dye from the moving floc was recorded by the CCD camera. In the settling test, the floc was transferred to the top of water in a settling column. The release of the dye from the floc during its settling became visible with the PIV laser illumination and was recorded by the PIV system. The mass transfer behavior of the AS flocs could be well demonstrated by the dye release from the flocs in a turbulent flow.

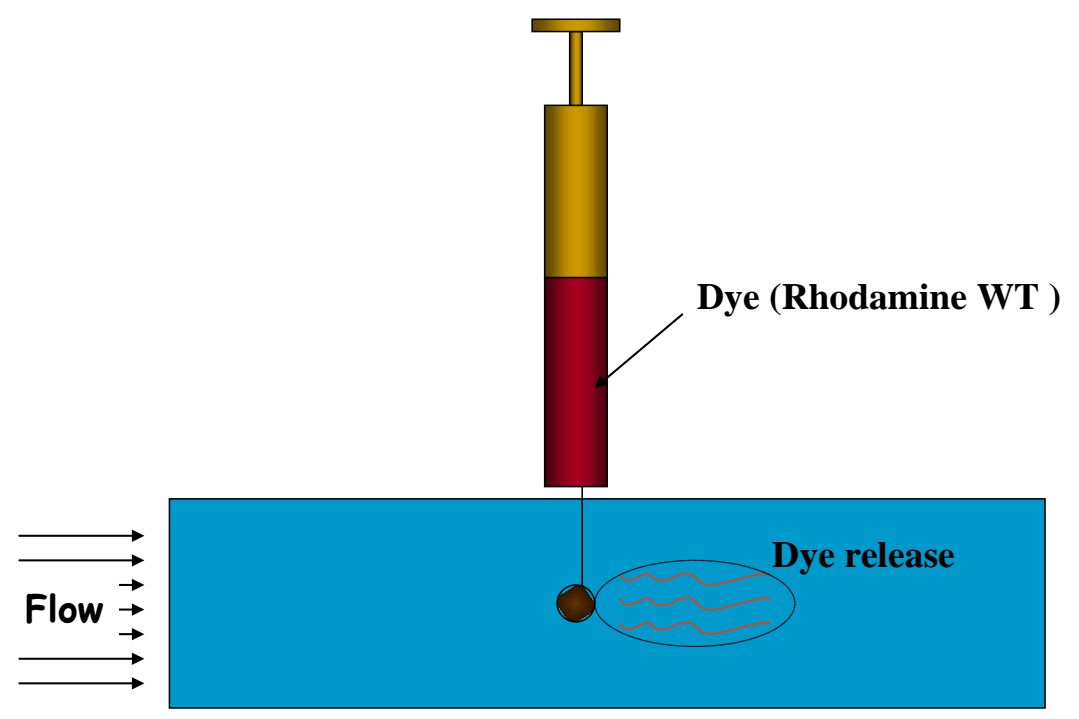

Fig. 2. Schematic of the dye release from the AS flocs in a turbulent flow test setup.

\section{RESULTS AND DISCUSSION}

The deformable feature of biological aggregates can be well observed from the PIV images. As the flow changed its direction, an AS floc in water changed its shape with the flow (Fig. 3). The fluid turbulence caused the shift of a few parts of the floc from one side to the other side in less than 200 milliseconds, which was captured by the PIV. It is apparent that, during the continuous shape change, the floc first shrank to a smaller volume. The floc then relaxed to a certain degree and swayed to a new shape. During this shape change process, the liquid within the floc would be forced out of the floc, which was then refilled with the liquid from the surrounding flow.

It is known that large aggregates are fractal objects that have an open and porous structure. The present PIV findings further indicate that fractal aggregates are not rigid particles. Instead, fractal aggregates have a soft and flexible shape that allows a considerable degree of deformation and shape change in water. This type of shape change and structural deformation would facilitate the exchange of fluid and materials between the interior of the aggregates and the surrounding water or solution. 

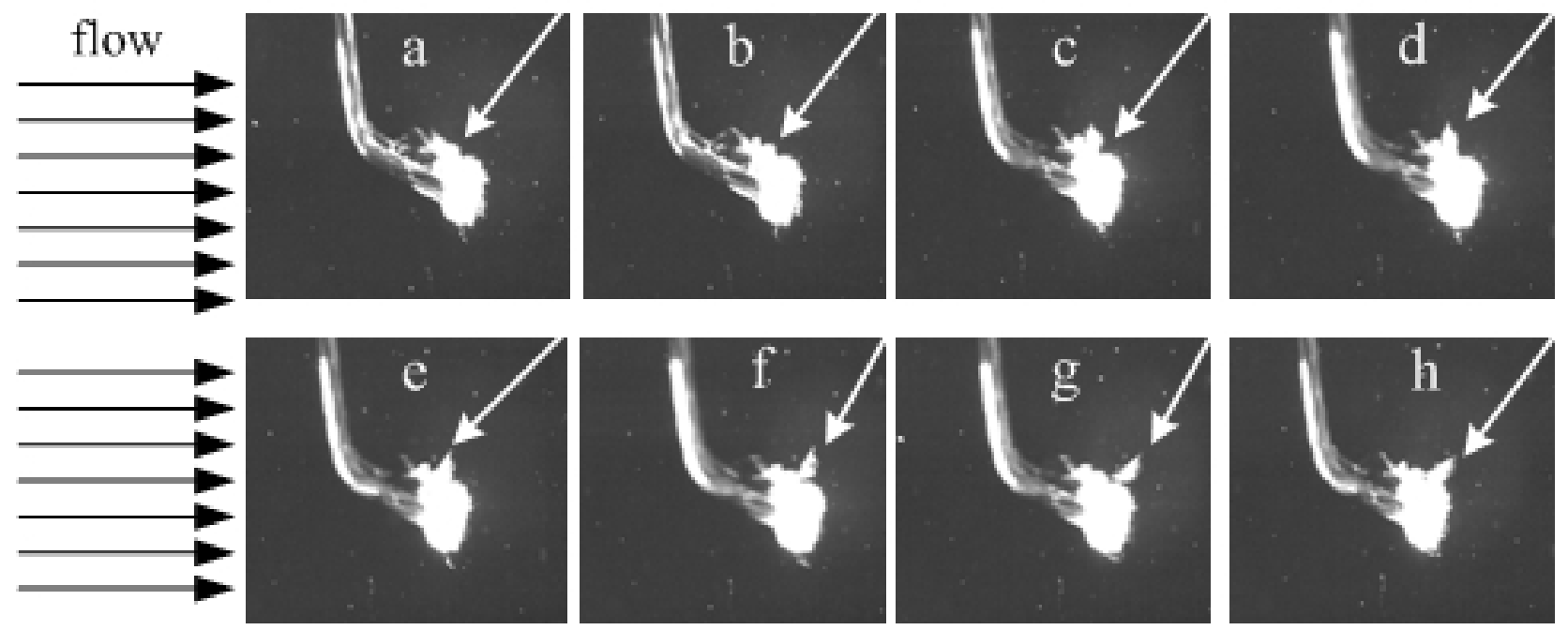

Fig. 3. PIV images for the shape change of an AS floc (with the arrow pointing to the main swaying part) in water during the change of the flow direction: images (a)-(h) were a series of photos of the floc that were taken by the PIV within 200 ms after the flow direction was reversed from the left to the right.

The release of dye from an AS floc during its settling in water was recorded in Fig. 4. The image indicates that, as the AS floc was porous and permeable, the dye inside the floc could release out with the internal flow through the floc. The release of the tracer dye during the settling of the floc however was rather stable and limited. This is likely due to the fixed and stable shape of the floc during its settling in the quiescent ambient water. However, the mass transport behavior of the flocs could be quite different when the flocs were moved forward and backward in water. As shown in Fig. 5, more dye could come off the flocs at a faster rate as the flocs had the shape changed with their movement. It is apparent that the floc shape change facilitated the dye release from the flocs and therefore enhanced the exchange of the fluid between the floc interior and the surrounding water.

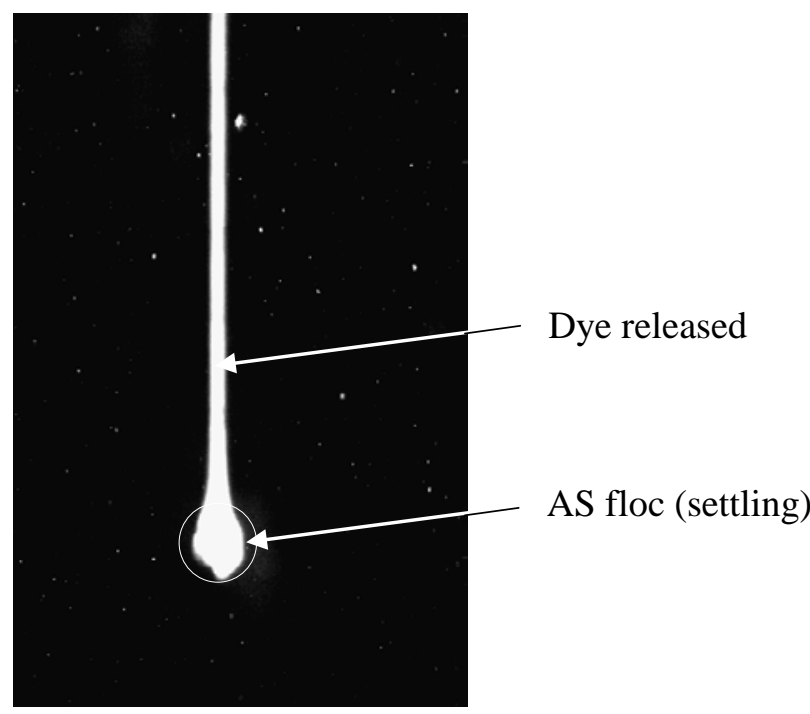

Fig. 4. PIV image for the dye release from an AS floc during its settling in water. 

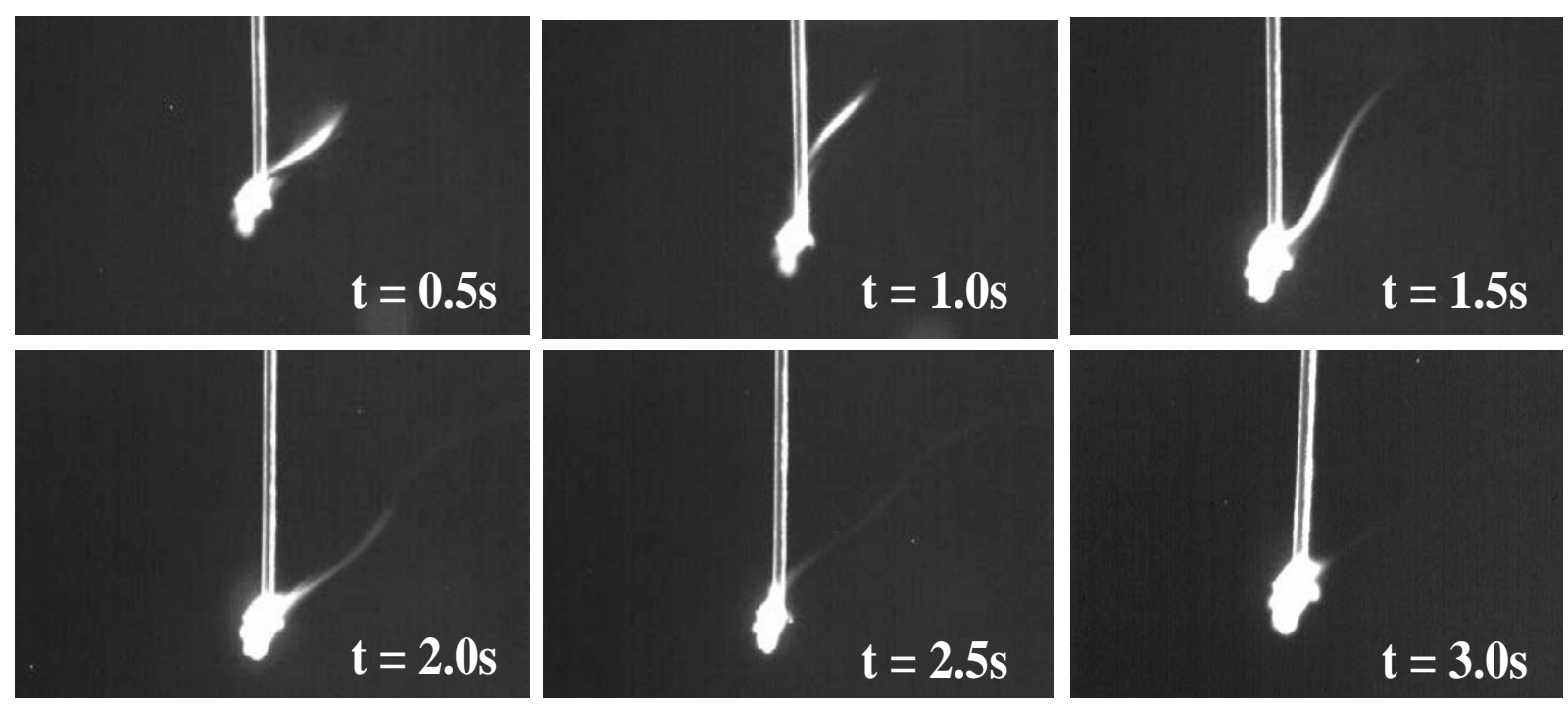

Fig. 5. PIV images for the dye release from an AS floc during its shape change in a turbulent fluid field.

The experimental results indicate that the mass transfer limitation for particle aggregates could be minimized considerably in a turbulent environment. Frequent shape change of the bio-flocs should be another mechanism of particle flocculation and mass transfer in biological wastewater treatment facilities. This particle and material transfer mechanism can be more important than molecular diffusion and internal permeation to the function and behavior of bio-flocs in natural waters and treatment processes. In addition, the frequent shape change would also affect the microenvironment within the bio-flocs, which has not been addressed previously. Moreover, the observed shape change of a floc is expected to enhance its aggregation with particles and cells in the suspension. However, the continuous deformation of the floc would weaken its structure and stability, leading to more breakages in a turbulent environment.

\section{CONCLUSIONS}

The shape change of AS flocs in water were directly characterized by the PIV technique. The results indicate that particle aggregates are soft and deformable fractal objects. The shape change of AS flocs in water flow would result in compression and relaxation of the flocs, forcing the liquid exchange between the interior of the flocs and the surrounding water. For the AS flocs saturated with a tracer dye, it was shown that the dye could release out of the flocs at a faster rate when the flocs were swayed around in water. It is argued that the deformation of bio-flocs can be another effective mechanism of material transport for flocs in biological wastewater treatment. This mechanism may be more important than molecular diffusion and internal permeation to the mass transfer of fractal bio-flocs in the turbulent fluid of natural waters and treatment systems.

\section{ACKNOWLEDGEMENTS}

This research was supported by grants HKU714811E from the Research Grants Council (RGC) and AoE/P-04/2004 from the University Grants Committee (UGC) of the Hong Kong SAR Government and project 51129803 from the Natural Science Foundation of China. The technical assistances of Mr. Keith C. H. Wong and Mr. C. H. Tong are greatly appreciated. 


\section{REFERENCES}

Clark M.M. (2003), Transport Modeling for Environmental Engineers and Scientists, Wiley, New York, USA.

Glasgow L.A. (2000). Floc deformation and expulsion of interstitial fluid. Environmental Technology, 21: 773-779.

Glasgow L.A. (2003). Deformation of individual aggregates and flocs. J. Dispersion Science and Technology, 24(5): 715-720.

Lam K.M. and Dai G.Q. (2002). Formation of vortex street and vortex pair from a circular cylinder oscillating in water. Experimental Thermal and Fluid Science, 26: 901-915.

Lam K.M. and Leung M.Y.H. (2004). Asymmetric vortex shedding flow past an inclined flat plate at high incidence. European Journal of Mechanics - B/Fluids, 24: 33-48.

Li X.Y. and Yuan Y. (2002). Collision frequencies of microbial aggregates with small particles by differential sedimentation. Environmental Science \& Technology, 36: 387-393.

Li X.Y., Yuan Y. and Wang H.W. (2003). Hydrodynamics of biological aggregates of different sludge ages: an insight into the mass transport mechanism of bioaggregates, Environmental Science \& Technology, 37, 292-299.

Raffel M., Willert C., Wereley S. and Kompenhans J. (2007). Particle Image Velocimetry: A Practical Guide, Springer, New York, USA.

Tao T., Peng X.F. and Lee D.J. (2006a). Interaction between wastewater-sludge floc and moving ice front. Chemical Engineering Science, 61: 5369-5376.

Tao T., Peng X.F., Lee D.J. and Hsu J.P. (2006b). Micromechanics of wastewater sludge floc: Force-deformation relationship at cyclic freezing and thawing. J. Colloid and Interface Science, 298: 860-868.

Xiao F., Lam K.M., Li X.Y., Zhong R.S. and Zhang X.H. (2011) PIV characterisation of flocculation dynamics and floc structure in water treatment. Colloids and Surfaces A: Physicochemical and Engineering Aspects, 379(1-3): 27-35.

Xiao F, Lam K.M. and Li X.Y. (2013) Investigation and visualization of internal flow through particle aggregates and microbial flocs using particle image velocimetry. J. Colloid and Interface Science, 397: 163-168.

Zhang J.J. and Li X.Y. (2003). Modeling particle-size distribution dynamics in a flocculation system. AIChE Journal, 49: 1870-1882. 